\title{
ENCUESTA DE OPINIÓN PARA LA EVALUACIÓN DELA GESTIÓN PÚBLICA EN COLOMBIA: UNA PROPUESTA DE MEDICIÓN*
}

\author{
Henry Antonio Mendoza Tolosa** \\ William Orlando Prieto Bustos ${ }^{* * *}$ \\ Carlos Alberto Barreto Nieto***
}

Recibido: diciembre 02 de 2011 • Aceptado: diciembre 13 de 2012

\section{RESUMEN}

El artículo propone implementar una encuesta de opinión cualitativa, dirigida a expertos, y con los resultados construir un índice orientado a evaluar la gestión de los gobiernos de manera continua, mediante una metodología que considera siete temáticas para analizar en un contexto diferente al financiero, la percepción sobre el desempeño institucional. El método abordado se basa en el estudio y adaptación en un cuestionario simplificado de las principales directrices fijadas por organismos multilaterales a los Estados para el seguimiento de sus responsabilidades y de las buenas prácticas estadísticas vigentes para el diseño de encuestas de percepción de opinión. El principal resultado obtenido es el desarrollo de un instrumento de evaluación de la gestión pública, soportado en referentes metodológicos internacionales, alternativo a la modelación financiera, útil para contrastar sus resultados e identificar el impacto de las políticas gubernamentales en la sociedad.

\section{PALABRAS CLAVE}

Gestión pública, encuestas de opinión, evaluación de desempeño.

\section{CLASIFICACIÓN JEL}

$\mathrm{Hl}$, H83, H89

\footnotetext{
Este artículo de investigación es producto del proyecto de investigación denominado "Índice de Gestión del Ejecutivo" del grupo de Política Económica de la Universidad Católica de Colombia y registrado formalmente ante COLCIENCIAS, el cual es resultado de una reflexión generada durante las sesiones del Comité de Investigaciones de la Facultad de Economía en el sentido de construir un conjunto de indicadores orientados a medir de manera permanente la gestión de los gobiernos a lo largo del tiempo con base en referentes aceptados en la literatura y que sean de amplia acogida internacional. Este proyecto fue financiado en su totalidad por la Universidad Católica de Colombia y fue ejecutado entre enero y noviembre del año 2011.

“ Economista y magíster en Ciencias Económicas, Universidad Nacional de Colombia, Bogotá, Colombia. Miembro del grupo de investigación de Política Económica, Universidad Católica de Colombia, Bogotá, Colombia. Profesor e investigador, Universidad Católica de Colombia. Coordinador de las Cuentas Nacionales Anuales del Departamento Administrativo Nacional de Estadística (DANE), Bogotá, Colombia. Correo electrónico: hamendoza@ucatolica.edu.co.

‥ Economista, Universidad Nacional, Bogotá, Colombia; máster en Políticas Públicas, Universidad de Rochester, New York, Estados Unidos. Miembro del grupo de investigación de Política Económica, Universidad Católica de Colombia. Profesor e investigador, Universidad Católica de Colombia, Bogotá, Colombia. Correo Electrónico:woprieto@ucatolica.edu.co.

.... Economista y magíster en Ciencias Económicas, Universidad Nacional, Bogotá, Colombia. Miembro del grupo de investigación de Política Económica, Universidad Católica de Colombia, Bogotá. Profesor e investigador, Universidad Católica de Colombia. Correo electrónico: cabarreto@ucatolica.edu.co
} 
Henry Antonio Mendoza Tolosa • William Orlando Prieto Bustos • Carlos Alberto Barreto Nieto

CONTENIDO

Introducción; 1. Metodología; 2. Resultados; 3. Conclusiones y agenda futura de trabajo; Bibliografía.

\section{OPINION SURVEY FOR ASSESSING PUBLIC MANAGEMENT IN COLOMBIA: A MEASUREMENT PROPOSAL}

\section{ABSTRACT}

This article proposes to implement a qualitative opinion survey aimed at experts and build and index based on the results orientated for continuously assessing governance using a methodology that aside from the financial aspect, considers other 7 different topics to be analyzed. The addressed method is based on the study and adaptation of a simplified questionnaire of the main guidelines set by multilateral agencies to Governments for tracking their responsibilities and for implementing the current statistical best practices in survey design for opinion perception. The main result is the development of an assessment tool for governance supported on international methodological referents different from financial modeling, useful to compare their results to identify the impact of government policies on societies.

\section{KEY WORDS}

Public management, opinion surveys, performance assessments.

\section{JEL CLASSIFICATION}

H1 1, H83, H89

\section{CONTENT}

Introduction; 1. Methodology; 2. Results; 3. Conclusions and future plans.

\section{PESQUISA DE OPINIÁO PARA A AVALIAÇÃO DA GESTÃO PÚBLICA EM COLÔMBIA: UMA PROPOSTA DE MEDIÇÃOO}

\section{RESUMO}

O artigo propõe aplicar uma pesquisa de opinião qualitativa, dirigida a expertos e com os resultados construir um índice orientado a avaliar a gestão dos governos de maneira continua, mediante uma metodologia que considera sete temáticas para analisar em um contexto diferente ao financeiro, a percepção sobre o desempenho institucional. O método abordado se baseia no estudo e adaptação em um questionário simplificado das principais diretrizes fixadas por organismos multilaterais aos Estados para o seguimento de suas responsabilidades e das boas praticas estatísticas vigentes para o desenho de pesquisas de percepção de opinião. O principal resultado obtido é o desenvolvimento de um instrumento de avaliação da gestão pública, suportado em referentes metodológicos internacionais, alternativo à modelação financeira, útil para contrastar seus resultados e identificar o impacto das políticas de governo na sociedade.

\section{PALAVRAS CHAVE}

Gestão pública, pesquisas de opinião, avaliação de desempenho

\section{CLASIFICACÃO JEL}

H1 1, H83, H89

\section{CONTEUDO}

Introdução; 1. Metodologia; 2. Resultados; 3. Conclusões e agenda futura de trabalho; Bibliografia. 
Encuesta de opinión para la evaluación de la gestión pública en Colombia: una propuesta de medición

\section{INTRODUCCIÓN}

Esta investigación se motivó en los trabajos publicados por el Instituto del BM y CNC (2002), INF (2009), Centro Nacional de Consultoría (2011) y Gallup (2011), entre otros, en los cuales se hacen valoraciones puntuales de la gestión del Presidente de turno, su grado de aceptación o popularidad, o bien, porque se concentraron en establecer la percepción que tiene la sociedad en el análisis puntual de un tema de interés (corrupción institucional, transparencia de la administración, popularidad o aceptación de los gobernantes, elecciones, etc.). Dichos estudios se efectuaron en su mayoría de forma independiente mediante consultas de opinión a la ciudadanía, sin cubrir de manera consolidada y frecuente la percepción de la comunidad sobre los temas centrales de la gestión del Gobierno en su conjunto y evaluar, así, el grado de eficiencia de las políticas institucionales y no de una sola figura que las representa.

En tal sentido, este trabajo propone implementar una encuesta de opinión de tipo cualitativo y con los resultados construir un índice orientado a evaluar la gestión de los gobiernos de manera continua, mediante una metodología que considera siete temáticas para analizar, en un contexto diferente al financiero, la percepción sobre el desempeño institucional del Ejecutivo.

Responder al objetivo formulado significa plantear una medida alternativa a la confusa variedad de cifras fiscales que, desde una óptica financiera definida por conducto legal, disfrazan la percepción de eficiencia en el cumplimiento de responsabilidades, y no expresan con propiedad la eficacia de la gestión pública en cuanto al debido impacto que deben generar en la comunidad los recursos asignados mediante decisiones de política.

Lahera (2004), Cardozo (2009), Torres (2007), Brawerman, Raggio y Ruiz (2007), Ospina (2000) y Prieto, Barreto y Mendoza (2011), entre otros, se constituyeron en referentes para el diseño de un instrumento de tipo cualitativo de opinión informada, que reúne la valuación de los aspectos centrales de la rama ejecutiva del poder público, como parte de una métrica orientada a constituir la base para mejorar su eficiencia respecto a la percepción de la sociedad, formular nuevas líneas de acción, reorientar las políticas públicas cuando la comunidad percibe que no van por el camino adecuado y servir como parámetro de control político de administraciones ineficientes o poco efectivas, lo que, en suma, significa promover prácticas de transparencia y buen gobierno frente a sus electores.

El principal resultado de este trabajo lo constituye el desarrollo de un instrumento de evaluación de la gestión pública alternativo a los basados en información financiera con el cual se puedan no solo contrastar sus resultados, sino identificar 
el impacto que han generado en la sociedad las políticas gubernamentales, de una manera continua y soportada en referentes metodológicos internacionales.

El artículo presenta, en primer lugar, la metodología abordada que incluye conceptos, referencias teóricas y empíricas que ilustran la construcción del instrumento, los criterios de análisis y el diseño de los diferentes capítulos de la encuesta. Asimismo, se plantean los lineamientos generales que dan lugar a la presentación de los resultados de la encuesta mediante la elaboración de un índice tipo Laspayres que, de manera consolidada, permite analizar la dinámica que tiene la opinión pública sobre la gestión del gobierno a lo largo del tiempo. En la segunda parte, apoyados en un ejercicio que simula los resultados de la encuesta para el periodo 2011-IV trimestre a 2014-III trimestre, se expone el esquema de tabulación y valoración de las respuestas y la construcción del índice, y se ejemplifica la serie de datos obtenidos en el lapso definido. Al final, se explican las principales reflexiones que la aplicación de una encuesta de opinión de esta naturaleza tendría en una mejor evaluación de la gestión pública.

\section{METODOLOGÍA}

Una encuesta de opinión de tipo cualitativo, orientada a ser resuelta periódicamente por expertos en tópicos de finanzas y administración del sector público, y cuyos resultados se consoliden en un índice cuyo propósito sea evaluar de manera habitual y a lo largo del tiempo la gestión de diferentes gobiernos, para analizar el cumplimiento de los objetivos que son responsabilidad del Estado, así como la percepción de la opinión informada sobre sus actividades habituales, constituye el eje central del diseño metodológico propuesto en este trabajo.

Por tanto, la primera parte de este capítulo aborda los referentes teóricos con templados en la investigación, que se basan en los lineamientos que los organismos multilaterales han definido para la evaluación y seguimiento de las políticas y las finanzas públicas, así como su ámbito de aplicación, la transparencia de los procesos de la Administración del Estado y la medición de aspectos críticos de la función pública desde una óptica ajena a la revisión de los balances fiscales e indicadores financieros que de ellos se derivan. En la segunda sección, se analizan los estudios de esta naturaleza aplicados hasta hoy en Colombia, los cuales evidencian su limitación en cuanto a un ejercicio integral de evaluación en este tema, y en tercer lugar, a partir de los antecedentes relacionados, presenta el diseño metodológico de la encuesta de opinión, el cual está influido de manera significativa por el marco legal colombiano sobre el que se sustenta el ejercicio cotidiano de las actividades del sector público. 
Encuesta de opinión para la evaluación de la gestión pública en Colombia: una propuesta de medición

\subsection{Referentes conceptuales}

La evaluación de la gestión de los gobiernos es un tema que ocupa la atención de muchos expertos, de los administradores del Estado e inclusive de los organismos multilaterales. Varios estudios se han desarrollado en busca de una línea metodológica adecuada para calificar las actividades del Estado, entre los que se destacan los de Bañon (2003), Guerrero (1995) y Ballart (1992) que, entre otros, han servido de referencia a dicho propósito.

No obstante, si bien es necesario considerar diferentes propuestas a la hora de implementar una metodología de evaluación del Ejecutivo, el principio fundamental de este tipo de instrumentos debe ser el que estén soportados en referentes metodológicos reconocidos en el ámbito internacional, de forma que tengan aplicación universal y no estén sujetos a ningún tipo de controversia teórica y subjetividad analítica. En tal sentido, el Fondo Monetario Internacional (FMI), organismo multilateral encargado de la formulación de la doctrina y directrices mundiales en materia de disciplina fiscal y buenas prácticas gubernamentales constituye el marco conceptual pertinente para este ejercicio.

En este sentido, una alternativa planteada por el FMI (2008) en este propósito la constituye evaluar la adopción de pautas de transparencia fiscal, mediante las cuales se definen parámetros de ética, valores de la Administración Pública y adopción de procedimientos confiables en el ejercicio de las actividades y dinámicas de las entidades públicas. Al respecto, el FMI (2008, p. 19) plantea una serie de pilares para la administración del Estado, entre los cuales se considera la definición de funciones y responsabilidades claras, la transparencia de los procesos presupuestales, el acceso irrestricto del público a la información del Estado y las garantías de integridad de los procedimientos a cargo del Gobierno. A partir de ellos, se definen pautas de evaluación de las actividades gubernamentales, de forma que se puedan comparar los resultados cualitativos de la gestión de los dirigentes de manera intertemporal, garantizando pautas de medición homogénea en los distintos momentos, así como consistencia y coherencia entre los diferentes instrumentos, datos fiscales y metas de política.

Otros lineamientos planteados por este organismo y considerados en el marco metodológico de este trabajo (FMI, 2001) se orientan a la formulación de parámetros de seguimiento y análisis fiscal estandarizados entre países, de forma que la evaluación de las finanzas públicas de los Estados acoja buena parte de las recomendaciones técnicas del Sistema de Cuentas Nacionales y demás metodologías de esta naturaleza, uniformes en los conceptos, así como reconocidas y adoptadas en el ámbito multilateral. El tercer grupo de recomendaciones adoptadas en la 
construcción de este ejercicio contempló los procedimientos estructurales para el diseño y construcción de indicadores financieros por parte del FMI (2006, p. 11), que para este organismo "constituye un hito en el establecimiento de una norma de referencia sobre los conceptos y las definiciones, las fuentes de datos y las técnicas relativas a la compilación y divulgación de indicadores de solidez financiera".

Por otra parte, la estructuración del esquema de medición de la gestión gubernamental en Colombia que se propone se ha apoyado también en las propuestas conceptuales formuladas por el DNP (2008) y el DANE (2010), mediante la divulgación de guías metodológicas para la elaboración de indicadores que se constituyan en herramientas prácticas para la evaluación de las responsabilidades públicas, ya que como lo afirma Lajera (2004, p. 8) "una política pública de calidad incluirá orientaciones o contenidos, instrumentos o mecanismos, definiciones o modificaciones institucionales y la previsión de sus resultados".

Haciendo alusión a un trabajo del Banco Mundial, el DNP (2008, p. 2) ha indicado que la evaluación de gestión constituye

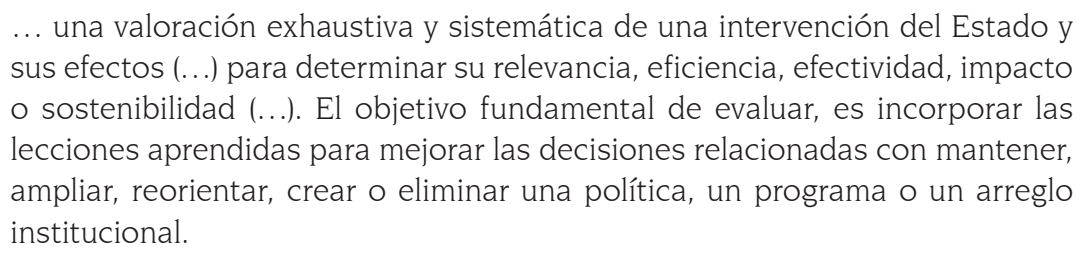
sus efectos (...) para determinar su relevancia, eficiencia, efectividad, impacto o sostenibilidad (...). El objetivo fundamental de evaluar, es incorporar las lecciones aprendidas para mejorar las decisiones relacionadas con mantener, ampliar, reorientar, crear o eliminar una política, un programa o un arreglo institucional.

El diseño de una encuesta de opinión para calificar la gestión del gobierno ha considerado las recomendaciones de los documentos metodológicos relacionados y en contexto, así como también se ha nutrido de diferentes trabajos estadísticos y de consulta de opinión que se han realizado en Colombia en diferentes períodos, orientados a analizar diversos aspectos de la función pública, algunos de los cuales serán comentados a continuación.

\subsection{Referentes de aplicación}

En el diseño de la Encuesta de Opinión fue muy significativo considerar diferentes experiencias que se han llevado a cabo en Colombia como referentes del instrumento a construir. En general, se detectó que dichos estudios se encaminaron a valorar temas específicos en un momento del tiempo y poco se interesaron por abarcar la evaluación de todas las dimensiones de acción del Estado. Así, la primera investigación que sirvió de referente al desarrollo del índice de gestión del ejecutivo con carácter cualitativo fue efectuada por el Instituto del BM (2002), la cual se basó en la valoración y seguimiento de las políticas de lucha contra la corrupción, 
Encuesta de opinión para la evaluación de la gestión pública en Colombia: una propuesta de medición

para establecer si las medidas adoptadas fueron consecuentes para controlar dicha práctica.

Los resultados de esta encuesta dejaron la percepción en Colombia de que la corrupción es un problema muy grave y las decisiones de política pública promueven, antes que evitar, las prácticas corruptas. Asimismo, la ciudadanía expresó inquietudes respecto a un efectivo proceso de rendición de cuentas por parte del Gobierno a tal punto que se concluyó que existen serias dudas sobre la transparencia, autocontrol, gobernabilidad e integridad del sistema político del país. Junto con esta, otras investigaciones de este tipo han llevado a concluir que los encuestados no confían en las entidades públicas porque perciben que no hay transparencia en los procesos de contratación que tienen a su cargo las entidades del Gobierno.

La firma de INF (2009, p. 29) realizó una encuesta que se basó en determinar una opinión general respecto a la imagen de los entonces candidatos a la presidencia, medir la intención de voto en la última contienda electoral, así como la opinión sobre los principales problemas que afectaban al país por esa época. En este último aspecto, el desempleo, el costo de vida, la inseguridad, la delincuencia común y la corrupción de la clase política centraron las preocupaciones de los potenciales electores.

La Misión de Observación Electoral y el Fondo de las Naciones Unidas para la Democracia (UNDEF, 2010, p. 16-17) diseñaron una investigación cuyo objetivo fue medir la percepción electoral de los votantes colombianos con base en una consulta de opinión relacionada con los aspectos de la agenda legislativa, los factores de decisión del voto local, los mecanismos de participación democrática y la confianza existente entre las entidades regionales sobre el proceso electoral, entre otros as pectos. Producto de la misma, se concluyó que las actividades proselitistas de los candidatos como el suministro de dadivas y la trashumancia electoral, entre otros problemas, afectan la transparencia del proceso electoral, el voto de opinión y la libre conciencia electoral del país.

Gallup (2011) y el CNC (2011) presentaron las investigaciones de opinión más recientes en este tema, las cuales tuvieron por objetivo evidenciar la percepción de los colombianos respecto al desempeño en las funciones del Gobierno nacional y la figura presidencial, en su papel frente a la corrupción, la economía, las relaciones internacionales y su labor en diferentes tópicos cuyos resultados ratificaron el mantenimiento de dificultades en dichos aspectos.

Los referentes conceptuales y las diferentes encuestas de opinión citadas, constituyeron los principales soportes para el diseño metodológico de la encuesta 
de tipo cualitativo para evaluar la gestión del ejecutivo en Colombia. En tal sentido, la siguiente sección describirá los criterios y componentes técnicos que se consideraron en su elaboración.

\subsection{Diseño metodológico de una encuesta de opinión al ejecutivo}

La primera inquietud por resolver consistió en dar respuesta a por qué se eligió en este caso como instrumento una encuesta de opinión dirigida a expertos y no otro mecanismo de evaluación. Sobre el particular, la investigación de la cual forma parte esta propuesta abordó de manera alternativa diseños de carácter cuantitativo y cualitativo para calificar la gestión de la Administración Pública.

Al respecto, los trabajos de Prieto, Barreto y Mendoza (2011) y Barreto, Mendoza y Prieto (2011) se enfocaron en el diseño de instrumentos de tipo cuantitativo o que están basados en dicha esfera de análisis. Las discusiones del grupo de investigación relacionadas con la importancia de disponer de una propuesta integral de medición de la gestión pública condujeron a incorporar el enfoque cualitativo como elemento de contraste de resultados y complemento de dichos estudios.

Dicho enfoque, tal como lo afirman Brawerman, Raggio y Ruiz (2007, p. 3) es apropiado "para dar cuenta de la resignificación de los objetivos de los programas por parte de los funcionarios y técnicos que los implementan, de sus percepciones y creencias" los cuales no se identifican con facilidad en un análisis de carácter financiero. AsImismo, tiene la ventaja de evidenciar las "vivencias y reacciones de los diferentes actores que participan de las intervenciones forman parte de las mismas y contribuyen a sus resultados", de manera que al ser contrastado con la información cuantitativa, es posible analizar en contexto el cumplimiento de las responsabilidades de los servidores públicos, sin depender exclusivamente de diagnósticos imprecisos cuyo origen son estadísticas financieras.

Hoy en día, el enfoque cualitativo que años atrás no era del todo aceptado como metodología alternativa tiene amplia difusión y aplicación, y en el caso particular de Colombia, los estudios citados son una buena muestra de su uso indistinto para hacer análisis sobre ciertos temas de interés que escapan al alcance de la órbita cuantitativa. Por todo lo anterior, el primer aspecto a considerar en el enfoque cualitativo de la Encuesta de Opinión sobre la Gestión al Ejecutivo (EGE) se consolidó en siete capítulos que compilan el conjunto de temas fundamentales para el ejercicio de la administración del Estado, al nivel de cada cartera, sector específico de la administración o su conjunto, a saber:

1. Ejecución presupuestal

2. Cumplimiento de metas

3. Contratación 
4. Impacto en la sociedad de las medidas

5. Eficiencia en la gestión y Administración Pública

6. Evolución del trámite de proyectos de interés

7. Relaciones internacionales

Los siete capítulos de la encuestal fueron definidos con base en la selección de los criterios de seguimiento más recurrentes postulados en los referentes metodológicos consultados, y en las diferentes encuestas e investigaciones empleadas como evidencia empírica. Así, entre los aspectos de transparencia promulgados por el FMI (2008), la planificación acertada (cumplimiento de metas), la eficiencia de la gestión y los procedimientos de contratación adecuados se destacan como los de mayor relevancia en su propuesta. Por su parte, algunas de las directrices fiscales más representativas que este organismo recomienda a los países miembros (FMI, 2001) consideran la ejecución del presupuesto y la medición estadística estandarizada de la gestión pública entre sus temas relevantes.

Finalmente, los capítulos relacionados con el impacto en la sociedad de las medidas, la evolución del trámite de proyectos de interés y las relaciones internacionales se seleccionaron por ser distintivos entre las diferentes encuestas aplicadas en Colombia y, en particular, por destacar puntos neurálgicos de la administración del Estado que no están concebidos con claridad en ninguno de los referentes teóricos trabajados que, en suma, contribuyeron a generar una propuesta que recoge de manera integral las cuestiones centrales a evaluar en la gestión pública.

Considerando este grupo de aspectos, se diseñó un cuestionario con un total de diez (10) preguntas (una o dos por temática) de valoración a escala, que abarcan cada uno de los capítulos mencionados y en las que el encuestado responde seleccionando el grado de acuerdo o desacuerdo frente a los temas específicos propuestos, permitiéndole, a su vez, contextualizar su respuesta de manera argumentada.

El cuestionario tiene por objeto consultar la opinión de expertos respecto a la percepción que tienen sobre el grado de cumplimiento de las responsabilidades de cada gobierno en cada sector administrativo de acción del Estado representado en las tareas asignadas a cada uno de los ministerios. A partir de la valuación de la gestión de cada cartera en los siete temas que cubre la encuesta, se pretende consolidar una evaluación conjunta del Gobierno nacional enfocada a determinar las fortalezas, debilidades y aspectos a mejorar en el ejercicio de la gerencia del Estado.

Por su parte, la definición de la muestra comprende, para período de estudio, la selección de un grupo de analistas, expertos en los temas relacionados con los

La encuesta diseñada se puede consultar en el anexo 
programas sectoriales y la agenda a cargo de cada ministerio, los cuales deben atender los siguientes criterios:

a. Los encuestados deben mantener independencia política y administrativa del Gobierno, de forma que no sean funcionarios de las entidades públicas consultadas y, de ser posible, que no pertenezcan a alguna entidad del Estado; tampoco deben sostener relación filial con alguna organización política del Gobierno o la oposición, ni mantener algún vínculo familiar o de consanguineidad con las altas direcciones de cada ministerio.

b. En segundo lugar, los encuestados deben ser expertos en los temas y agendas propias de la cartera específica por la cual se les consulta su opinión, bien porque han desempeñado en alguna ocasión cargos relacionados con las responsabilidades del sector consultado o porque tienen una amplia trayectoria en la investigación de la problemática de dicho sector.

c. Finalmente, los expertos deben ser reconocidos por la opinión pública, bien, por su prestancia académica o su profesionalismo, o por la objetividad con que plantean inquietudes en diferentes escenarios.

La decisión de aplicar la encuesta a expertos en lugar de hacerlo mediante una consulta a la ciudadanía reside en que los temas que esta cubre abordan aspectos especializados de la actividad del sector público sobre los que normalmente el ciudadano del común no está informado, o lo está pero en forma no adecuada o incompleta, pues es posible que pueda tener información sobre el desempeño de alguna(s) carteras pero difícilmente de todas y, finalmente, porque su percepción sobre dichos aspectos podría estar influida en la mayoría de los casos por las opiniones expresadas en los medios de información a los que tiene acceso habitualmente.

En tal caso, obtener un grado apropiado de objetividad y de percepción de opinión en las respuestas de un cuestionario que es especializado en sus contenidos se garantiza en mayor medida teniendo como requisito el que la encuesta sea respondida por personajes que conocen con suficiencia los temas de la agenda y la dinámica de cada ministerio, antes que por ciudadanos del común. Por tanto, en la selección de los expertos sería conveniente designar para cada cartera un grupo de tres a cinco personalidades que cumplan los criterios definidos, seleccionando para su postulación a un grupo de representantes de la academia o de alguna organización no gubernamental ajena a los intereses del Gobierno. Asimismo, en la medida que la aplicación de la encuesta se prevé que tenga periodicidad trimestral y se garantice precisión en la percepción de opinión, será prudente en un contexto estadístico y analítico cambiar con cierta regularidad a alguno(s) experto(s) consultados por cada ministerio. 
Encuesta de opinión para la evaluación de la gestión pública en Colombia: una propuesta de medición

En tal sentido, el cuerpo de la EGE en su primer capítulo aborda el tema de la Ejecución Presupuestal y busca obtener una evaluación de los expertos consultados en torno a la efectividad de la gestión presupuestal de cada ministerio de conformidad con las responsabilidades asignadas y con la programación de gastos de cada vigencia, preparada y disponible de manera oportuna. Esta valoración puede diferir del resultado fiscal según el estatuto orgánico del presupuesto, en la medida que si bien el registro de las diferentes etapas de la cadena de ejecución presupuestal puede acoger lo reglamentado en esta norma, ello no significa que este cumpla con los propósitos contemplados de gasto.

Lo anterior quiere decir que una disciplinada programación de las disponibilidades presupuestales, los compromisos, las obligaciones, los pagos e, inclusive, las reservas de ley no es garantía suficiente para afirmar que el gobierno hizo las compras que debía hacer o que los gastos a cargo de cada Ministerio se ejecutaron en forma adecuada para atender las demandas sociales y mejorar el bienestar general ${ }^{2}$.

El segundo capítulo de la EGE relacionado con el Cumplimiento de Metas tiene como propósito evaluar los resultados de la planeación de cada Ministerio de conformidad con el avance de las metas gubernamentales propuestas por el Ejecutivo en cada sector específico durante el período analizado, a partir de una batería de indicadores específicos de gestión a los cuales el Departamento Nacional de Planeación les hace seguimiento habitual o, bien, porque identifica con claridad los bienes y servicios entregados a la sociedad en los plazos pactados acorde con la programación de sus actividades. Asimismo, la ejecución del presupuesto al guardar una estrecha relación con las metas formuladas en el Plan Nacional de Desarrollo debe reflejar avances físicos, observables, cuantificables de corto y mediano plazo coherentes con los productos previstos en su planeación de largo plazo, que sean trazables, transparentes y acordes con los propósitos de gestión planteados allí por el Gobierno nacional.

En tercer lugar, la EGE abarca el tema de la Contratación de la Administración Pública alrededor del cual surgen dos perspectivas de análisis. La primera, relacionada con la evaluación de la transparencia y calidad de los procesos de contratación en cada cartera, y la segunda, con la valoración del avance de los productos y servicios contratados.

En el primer caso, se pretende analizar la dinámica de la contratación como pilar de la gestión administrativa de los diferentes ministerios, así como la atención de los

2 En relación con las orientaciones a seguir por los gobiernos en materia presupuestal el Fondo Monetario Internacional (FMI) ha planteado diferentes referentes de orientación a la práctica presupuestal como el Manual de Transparencia Fiscal (2008), los Indicadores de Solidez Financiera (2006) y el Manual de Estadísticas de las Finanzas Públicas (2001), entre los más representativos.

Semestre Económico, volumen 15, №. 32, pp. 77-102 • ISSN 0120-6346, julio-diciembre de 2012, Medellín, Colombia 
conductos regulares contemplados para los procesos de contratación reglamentados por las Ley 80 de 1993 (CRC, 1993) y Ley 1150 de 2007 (CRC, 2007). De otro lado, se busca obtener la opinión de los expertos consultados para que evalúen el progreso alcanzado en cada período de los servicios y productos contratados de forma que se constituyan como elemento complementario de coherencia de la encuesta en sí mismos, así como de consistencia programática entre las metas formuladas en el plan de desarrollo y la ejecución del presupuesto, analizadas en los dos primeros capítulos de la EGE.

En el siguiente aparte, se analiza el Impacto en la Sociedad de las Medidas adoptadas por el Gobierno, capítulo que consta de dos secciones: la primera, orientada a determinar el impacto social de las medidas y acciones del Gobierno sobre la comunidad con independencia de la percepción general de las personas, dado que es natural que muchas medidas gubernamentales cuya finalidad sea de interés colectivo no sean populares entre muchos miembros de la comunidad, verbigracia toque de queda, nuevos impuestos específicos, etc. En este mismo sentido, el análisis del efecto generado en la sociedad con las políticas públicas debe evaluarse con independencia de su costo fiscal.

Como complemento a este análisis, la segunda sección del cuarto capítulo de la EGE consulta el grado de aceptación que tienen los ciudadanos ante las medidas de política implementadas por el Gobierno en cada sector específico durante el período analizado, desde la perspectiva de los expertos consultados. Si bien la pregunta del cuestionario no es planteada de forma explícita a la sociedad, se espera que la opinión informada del analista consultado en cada sector específico sea un referente objetivo que recoja el sentimiento popular del grado de aceptación de las políticas públicas.

El quinto componente de la EGE aborda el concepto de eficiencia en la gestión y Administración Pública, y tiene como objetivo disponer de una evaluación cualitativa general del conjunto de actividades realizadas en cada cartera por sector específico durante el período analizado. En términos prácticos, esta sección recoge la opinión consolidada de todos los demás capítulos de la encuesta, a partir de la inspección global de los criterios técnicos, administrativos, presupuestales, de control y los ámbitos político, económico y social del Gobierno general. Los resultados obtenidos en este módulo serán empleados para contrastar el conjunto de las respuestas de las demás secciones de la encuesta en cada ministerio.

La sección sexta de la EGE comprende el análisis sobre la evolución del trámite de proyectos de interés que durante el período evaluado haya o no efectuado el Gobierno de forma que pueda determinarse la oportunidad así como el avance 
Encuesta de opinión para la evaluación de la gestión pública en Colombia: una propuesta de medición

en el trámite de normas relacionadas con las metas y propósitos del ejecutivo en cada sector administrativo. De conformidad con los parámetros de un Estado de derecho, las actividades públicas están sustentadas en las normas expedidas, las cuales soportan la legalidad de las decisiones y legitiman el respaldo popular a las medidas de política. En este sentido, una restricción considerable para las medidas de política gubernamentales está dada por la sujeción del Ejecutivo al trámite de leyes y/o normas que dependen de los procedimientos que son responsabilidad de otras ramas del poder público o por instancias administrativas de otros niveles del Estado, por lo cual se generan atrasos que interrumpen de manera significativa la eficiencia pública.

El último componente de la EGE corresponde a la evaluación de las Relaciones Internacionales, el cual presenta dos ópticas de análisis: en la primera, se revisa cómo impactan las medidas gubernamentales de cada sector específico el manejo de las relaciones diplomáticas del país con Estados vecinos y no vecinos. Sin embargo, es preciso recordar que no todas las actividades de las carteras tienen repercusión directa o indirecta, voluntaria o involuntaria en las relaciones con otros países, en la medida que buena parte de las medidas de política se basan en fundamentos técnicos, antes que políticos o geoestratégicos.

En razón de lo anterior, la segunda perspectiva tiene por finalidad indagar el impacto técnico medido a través de los canales de cooperación propiciados con otros países en los temas que son responsabilidad de cada ministerio. Este tema adquiere importancia en razón a que, para citar un ejemplo, uno de los criterios de acceso de Colombia como miembro activo de la Organización de Estados para la Cooperación y el Desarrollo Económico -OCDE- es que el país sea prestador neto antes que perceptor neto de cooperación y asistencia técnica respecto a otros países. En todo caso, se ha considerado que en este módulo de la EGE, cualquiera de estas dos alternativas o ambas no solo se presentan habitualmente sino que están asociadas con las relaciones internacionales del Estado en su conjunto3.

Con base en lo anterior, y una vez adelantado el proceso de aplicación de las EGE, las respuestas tendrán asociadas en cada capítulo una valoración nominal a escala, cuyo puntaje acumulado para el conjunto de los ministerios dará lugar a la construcción de un índice que recoja a lo largo del tiempo la evolución y dinámica de la percepción sobre la gestión del Gobierno en cada período. Este índice, denominado Índice de Gestión del Ejecutivo (IGE), se constituirá en un indicador de referencia para evaluar a corto, mediano y largo plazo el desempeño de los diferentes

\footnotetext{
${ }^{3}$ A partir de la descripción hecha y para su consulta, la versión final de la encuesta consolidada se presenta en el Anexo 1 de este documento.
}

Semestre Económico, volumen 15, №. 32, pp. 77-102 • ISSN 0120-6346, julio-diciembre de 2012, Medellín, Colombia 
ministerios de la rama ejecutiva del Gobierno nacional en los aspectos fundamentales de la Administración Pública; asimismo se constituirá en un termómetro para los mandatarios de turno a la hora de analizar la orientación de las políticas públicas y la percepción que tenga la comunidad sobre la eficiencia de su gestión. En la siguiente sección, se presenta, a manera de ejemplo, un ejercicio simulado con datos hipotéticos mediante los cuales se construye una serie del índice, con el objetivo de servir de ilustración a los lectores sobre su aplicación y utilidad.

\section{RESULTADOS}

En primer lugar, la encuesta de opinión a expertos debe conducir a una evaluación global de la gestión del ejecutivo en cada período analizado (se propone que la encuesta sea trimestral). Para tal efecto, en el proceso de tabulación de resultados, cada una de las respuestas tiene una puntuación definida por la importancia que tiene cada componente o capítulo de la encuesta en la gestión de la cartera evaluada y que oscila entre cero (0) y dos (2) puntos, en el que (0) representa que el criterio no se cumple a cabalidad y (2) que se cumple a satisfacción, con un punto central (1) en referencia a un criterio o aspecto de la administración que se cumple de manera relativa.

Por ello, aspectos como el presupuesto, la planificación, la contratación y el impacto social de las medidas tienen el mayor peso en la evaluación con una valoración de hasta dos (2) puntos, en virtud a la trascendencia que estos temas tienen en la percepción general de eficiencia y eficacia de un gobierno a partir de los criterios definidos por el FMI (2008). Por su parte, los capítulos correspondientes al trámite de proyectos de interés y las relaciones internacionales, por tener un impacto secundario entre la opinión a la hora de evaluar la gestión pública, les fue asignada una puntuación que oscilará entre cero (0) y uno (1), de forma que en suma se consolide en cada periodo y para cada ministerio una evaluación general de las actividades bajo su responsabilidad que oscilará en un rango de cero (0) a diez (10) puntos.

Sin embargo, en la medida que la encuesta es de opinión, el rango definido para la calificación de las respuestas no considera una amplia variedad de percepciones (puntuaciones), dado que lo se pretende es concretar una evaluación conjunta que defina con claridad en cada sector específico y para cada temática de la gestión, el desempeño bueno, malo o regular del Gobierno.

Es ampliamente conocido que muchas investigaciones estadísticas contemporáneas cuantitativas o cualitativas tienen preguntas que son empleadas para confirmar la consistencia de algunas respuestas. En el caso de la EGE el componente 
Encuesta de opinión para la evaluación de la gestión pública en Colombia: una propuesta de medición

de "Eficiencia" calificado por los expertos entre cero (0) y dos (2) puntos, actúa de manera independiente frente a las demás preguntas del formulario y no se tiene en cuenta en la suma de resultados de los demás capítulos, en la medida que, como se explicó, se tomará como pregunta de contraste en la percepción de gestión de los expertos en cada ministerio, tal como se ilustra en el ejercicio simulado que se preparó para tal fin (ver cuadro 1) para los datos consolidados de un trimestre (2011-IV en el ejemplo).

En este caso, el criterio de eficiencia global se contrasta con la suma del puntaje asignado a las respuestas de cada capítulo de la EGE por Ministerio. Por tanto, si en este capítulo la calificación es igual a cero (0) y la suma de las demás se encuentra en un rango entre cero (0) y tres (3), la gestión de dicha cartera se considerará Ineficiente. Por otra parte, si Eficiencia es igual a uno (1) y la suma de las respuestas complementarias se encuentra en un rango de cuatro (4) a siete (7), la gestión del ministerio respectivo se considerara como Relativamente Eficiente. Finalmente, si eficiencia es igual a dos (2) puntos y se contrasta con la suma de las demás respuestas en un rango de ocho (8) a diez (10), la gestión del ministerio se calificara como Eficiente. En todo caso, la valuación de la pregunta de contraste no invalidará la calificación de los demás módulos de la encuesta, pues como se ha explicado, se empleará solo para confirmar las percepciones de los encuestados y en todo caso ni su tabulación, ni los criterios de puntuación serán de conocimiento de los encuestados, pues serán de aplicación exclusiva por parte de quien opere como administrador de la EGE.

Cuadro 1. Encuesta de Gestión al Ejecutivo: Tabulación de resultados periodo base (Ejercicio de Simulación para 2011-IV trimestre)

\begin{tabular}{|c|c|c|c|c|c|c|c|c|c|c|c|c|c|c|c|c|c|}
\hline $\begin{array}{l}\text { Categoría de } \\
\text { evaluación }\end{array}$ & 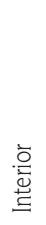 & 尊 & 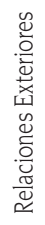 & 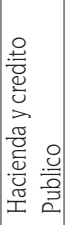 &  & 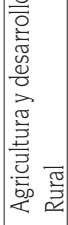 & 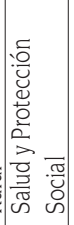 & 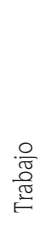 & 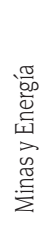 & 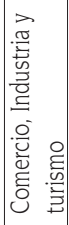 &  &  & 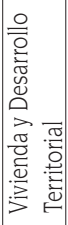 &  & 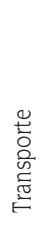 & $\frac{\text { 营 }}{3}$ &  \\
\hline Presupuesto & 2 & 2 & 1 & & & 0 & 2 & 2 & & 1 & 2 & 1 & 1 & 1 & 1 & 0 & 1,3 \\
\hline Metas gobierno & 1 & 1 & 2 & & & & 1 & 1 & & 0 & 1 & 2 & 2 & 2 & 0 & 1 & 1,2 \\
\hline Contratación & 0 & 0 & 0 & & & & 0 & 0 & & 1 & 2 & 0 & 0 & 2 & 0 & 1 & \\
\hline $\begin{array}{l}\text { Impacto social de las } \\
\text { medidas }\end{array}$ & 2 & 2 & 2 & & & & 1 & 1 & & 1 & 0 & 1 & 1 & 2 & 1 & 1 & 1,4 \\
\hline
\end{tabular}




\begin{tabular}{|c|c|c|c|c|c|c|c|c|c|c|c|c|c|c|c|c|c|}
\hline $\begin{array}{l}\text { Categoría de } \\
\text { evaluación }\end{array}$ & 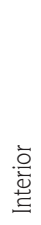 & 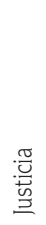 &  & 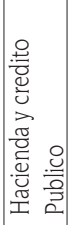 & 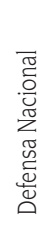 & 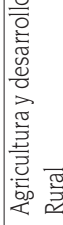 &  & 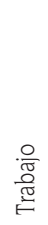 & 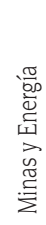 &  & 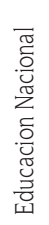 & 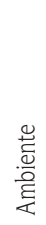 & 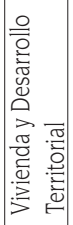 & 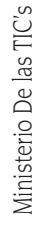 & 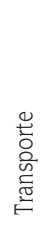 & $\frac{\text { 营 }}{3}$ & 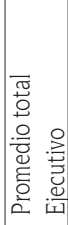 \\
\hline Trámite proyectos & 1 & 1 & 1 & & & & 1 & 1 & & 1 & 1 & 1 & 1 & 1 & 0 & 0 & 0,9 \\
\hline $\begin{array}{l}\text { Relaciones } \\
\text { Internacionales }\end{array}$ & 1 & 1 & 1 & & 1 & 1 & 1 & 1 & 1 & 1 & 1 & 1 & 1 & 1 & 1 & 0 & 0,9 \\
\hline TOTAL & 7 & 7 & 7 & 8 & 6 & 6 & 6 & 6 & 10 & 5 & 7 & 6 & 6 & 9 & 3 & 3 & 6,4 \\
\hline Eficiencia & 2 & 2 & 2 & 2 & 1 & 1 & 1 & 1 & 2 & 1 & 2 & 1 & 1 & 2 & 0 & 0 & 1,3 \\
\hline
\end{tabular}

Fuente: Ejercicio de simulación de resultados para el periodo 2011-IV.g

El ejercicio hipotético del cuadro 1 constituye una matriz de evaluación de resultados de la gestión de la rama ejecutiva, pues en ella se consigna la puntuación total de cada ministerio y por cada uno de los componentes de la encuesta (columnas); asimismo, el cuadro consolida la calificación media del Gobierno en cada capítulo de la misma (filas). Es así como para el total de la rama ejecutiva la evaluación global promedio de todos los ministerios para el periodo base (2011-IV trimestre) fue seis punto cuatro $(\mathbf{6}, \mathbf{4})$, la cual es coherente en comparación con el referente promedio de Eficiencia obtenido $(\mathbf{1}, \mathbf{3})$. Asimismo, los resultados consolidados permiten anali zar la evaluación total promedio del Gobierno central en cada uno de los capítulos temáticos de la encuesta, en la que la ejecución del presupuesto obtuvo el mejor puntaje medio $(\mathbf{1}, \mathbf{3})$ y la contratación el más bajo $(\mathbf{0 , 7})$.

Los resultados de la puntuación asignada al total del Gobierno se utilizarán para construir un índice tipo Laspayres ${ }^{4}$ en el cual se pueden comparar los cambios de evaluación en la gestión del Ejecutivo así como la tendencia que sigue la calificación de un gobierno o de diferentes administraciones a corto, mediano y largo plazo. Por tanto, el cuadro 2, ilustra cómo a partir de los resultados obtenidos en el periodo base (2011-IV) más los que se generarían siguiendo el mismo protocolo de la EGE hasta el tercer trimestre de 2014 se proyecta trimestre a trimestre el resultado del índice de gestión del Ejecutivo, incrementando el índice de cada vigencia con la variación porcentual de la evaluación de cada periodo respecto al inmediatamente anterior.

${ }_{4} \quad$ Un índice Laspayres es un número que muestra la relación estadística de cambio de una variable con referencia a un periodo específico definido como punto de partida o base. 
Encuesta de opinión para la evaluación de la gestión pública en Colombia: una propuesta de medición

Cuadro 2. Encuesta de gestión al Ejecutivo:

Construcción del Índice Tipo Laspayres, Serie 2011-IV a 2014-III

Base 2011 -IV trimestre $=100$

\begin{tabular}{|c|c|c|}
\hline Trimestre & Evaluación & Proyección índice \\
\hline 2011 -IV & 6,4 & 100,0 \\
\hline $2012-1$ & 6,7 & 104,7 \\
\hline $2012-11$ & 7,3 & 114,1 \\
\hline $2012-$ III & 7,0 & 109,4 \\
\hline $2012-$ IV & 7,1 & 110,9 \\
\hline $2013-1$ & 6,8 & 106,3 \\
\hline
\end{tabular}

\begin{tabular}{|c|c|c|}
\hline Trimestre & Evaluación & Proyección índice \\
\hline $2013-11$ & 6,5 & 101,6 \\
\hline $2013-$ III & 6,0 & 93,8 \\
\hline $2013-$ IV & 5,8 & 90,6 \\
\hline $2014-1$ & 5,6 & 87,5 \\
\hline $2014-11$ & 6,2 & 96,9 \\
\hline $2014-$ III & 6,8 & 106,3 \\
\hline
\end{tabular}

Fuente: Simulación de resultados consolidados de la evaluación de la EGE

En la simulación efectuada, se aprecia cómo el punto más alto de la calificación del ejecutivo se presenta en el segundo trimestre de 2012 cuando la evaluación fue de 7,3 puntos (índice 114,1) y el punto más bajo inferior en el primer trimestre de 2014 con 5,6 puntos (índice 87,5). Finalmente, el gráfico 1 presenta la dinámica del Índice de Gestión al Ejecutivo a lo largo de la serie, así como la tendencia que siguió la percepción de los expertos sobre la gestión del Gobierno.

Gráfico 1. Índice de gestión del Ejecutivo, 2011 -II a 2014-III

Índice Laspayres, base diciembre de $2011=100$

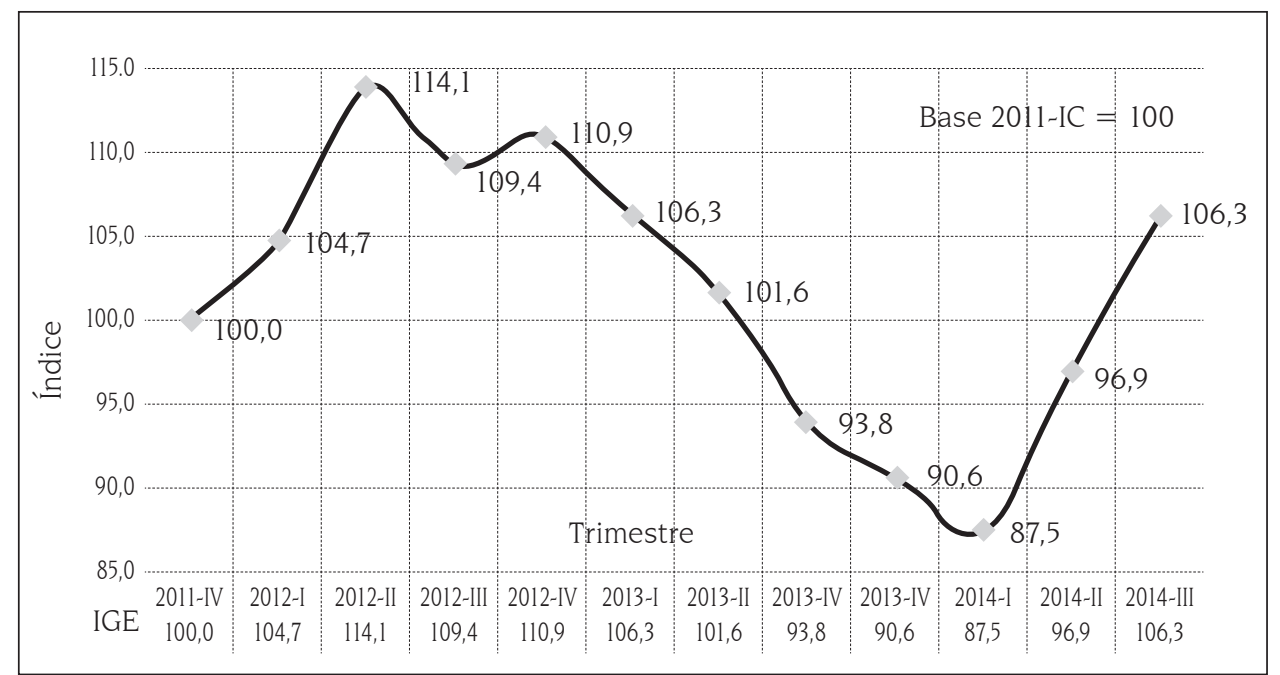

Fuente: Elaboración autores con base en datos simulados 


\section{CONCLUSIONES Y AGENDA FUTURA DE TRABAJO}

La formulación de un Índice de Gestión del Ejecutivo -IGE- puede materializarse desde diferentes enfoques metodológicos, los cuales, en su mayoría, tienen como base de cálculo los resultados contables y financieros del sector público. Sin embargo, una nueva alternativa por explorar surge de la valuación cualitativa de las actividades ejecutadas en el corto plazo por el Gobierno nacional, mediante el diseño, aplicación y consolidación de resultados de una Encuesta de Opinión cuya base conceptual contiene reconocidas metodologías de frontera aceptadas en el ámbito mundial para el diseño de indicadores de eficiencia de las políticas públicas de corto plazo. Dicho instrumento debe ser diligenciado período a período por un grupo de expertos en finanzas públicas y administración del Estado en cada sector administrativo, seleccionados cuidadosamente por un grupo respetado de académicos o una organización sin vínculos políticos con el gobierno de turno, para que expresen su opinión sobre la gestión de la rama ejecutiva del sector público, en cada cartera ministerial, de manera periódica.

Una evaluación cualitativa del Gobierno se justifica en razón a que esta propuesta permite analizar aspectos particulares de la gestión pública como la percepción de acogida de las medidas públicas o los niveles de corrupción administrativa, que con escasa probabilidad se evidencian en la lectura de las estadísticas fiscales difundidas. Hoy en día, este tipo de análisis constituye un requerimiento recurrente por parte de los organismos multilaterales, que como el FMI (2006, p. 13), indican cómo es de importante disponer de un conjunto de indicadores oportunos que midan la efectividad de las diferentes decisiones de política.

Mediante un ejercicio simulado con fines ilustrativos se recrearon y puntuaron resultados hipotéticos de la encuesta, se consolidó una evaluación media de la gestión del Gobierno en general, de cada ministerio en particular y de cada uno de los aspectos centrales de análisis de la administración del Estado. Asimismo, para analizar de forma estandarizada los resultados consolidados de la EGE se elaboró un índice tipo Laspeyres que representa la dinámica que sigue a lo largo del tiempo la evaluación de la gestión del Gobierno, de forma que se utilice como instrumento de toma de decisiones y reorientación de políticas por parte del Estado así como herramienta de control ciudadano y de conciencia electoral de la comunidad en general.

En este sentido, la agenda futura de investigación en este campo debe abordar la definición del administrador de la EGE y programar bajo su responsabilidad la implementación de una prueba piloto por lo menos durante tres períodos consecutivos, en la que se aplique el formulario a un grupo de reconocidos expertos que conduzca a ajustes pertinentes del cuestionario, analizar la metodología de tabu- 
Encuesta de opinión para la evaluación de la gestión pública en Colombia: una propuesta de medición

lación de respuestas, analizar en contexto los resultados y determinar el trimestre de referencia o base del índice.

Asimismo, nuevos trabajos sobre esta propuesta deben orientarse a integrar, combinar, analizar y describir los resultados de la Encuesta de Gestión al Ejecutivo en conjunto con las propuestas de Barreto, Mendoza y Prieto (2011) y Prieto, Barreto y Mendoza (2011) presentadas como alternativas de esta línea de investigación, de manera que puedan articularse en un sistema integrado de evaluación de gestión al ejecutivo que combine el análisis cuantitativo y cualitativo de la Administración Pública en Colombia en un horizonte continuo. Asimismo, la construcción de sistemas de indicadores integrados de gestión para los gobiernos locales basados en metodologías aceptadas en el contexto internacional, mediante los cuales se puedan definir protocolos estandarizados de evaluación de la administración territorial han sido considerados en la agenda.

En suma, la implementación de la Encuesta de Gestión al Ejecutivo como un instrumento alternativo de percepción de opinión para evaluar los gobiernos complementa el análisis de la gestión de la Administración Pública desde una óptica diferente a la financiera y permite contrastar de forma continua y con rigurosidad, mediante un índice que consolida las respuestas tabuladas, la valoración ambigua que ofrecen las estadísticas fiscales de la Administración Pública.

\section{BIBLIOGRAFÍA}

Ballart, Xavier (1992). ¿Cómo evaluar programas y servicios públicos?: aproximación sistemática y estudios de caso. Ministerio para las administraciones públicas. [En línea]. Secretaria Técnica General. Instituto Nacional de Administración Pública. 284p. Primera edición Mayo 1992. Disponible en línea en http://books.google.com/books?id=HEAfcrVGjZoCEprin


false. [Consultado en agosto de 2011].

Bañón, Rafael (2003). La evaluación de la acción y de las políticas públicas. [En línea]. Madrid, España. Ediciones Díaz de Santos. 253p. Disponible en http://books.google.com/ books?id=9OE4SRJV-HUCEprintsec $=$ frontcoverEhl=esEsource $=$ gbs _ ge _ summary $\mathrm{rEcad}=0 \# \mathrm{v}=$ onepageEqEf=false. Madrid - España. [Consultado en agosto de 2011].

Barreto Carlos, Mendoza Henry, Prieto William (2011). Un índice de concentración del presupuesto público. Documento de trabajo. En Mimeo, Universidad Católica de Colombia, Facultad de Economía. p. 25.

Brawerman, Josette; Raggio, Liliana; Ruiz, Violeta (2007). Abordajes Cuanti-Cualitativos en la Evaluación de Programas desde la Gestión Estatal. Ponencia presentada al IV 
Congreso Argentino de Administración Pública. Secretaría de Gestión Pública. Asociación de Administradores Gubernamentales. AAEAP. [En línea]. Buenos Aires, 22-24 de agosto de 2007. Editorial: Fundación CRISOL. Buenos Aires, Argentina. Disponible en http:// ceadel.org.ar/centrodoc/?p=229. [Consultado en abril 2012].

Cardozo Brum, Myriam (2009). La institucionalización de una cultura de la evaluación en la Administración Pública mexicana: Avances y desafíos pendientes. [En línea]. En Convergencia, Revista de Ciencias Sociales. Universidad Autónoma del Estado de México. Toluca, México. Vol. 16, Núm. 49, enero-abril, 2009, pp. 175-198. Disponible en línea en http://redalyc. uaemex.mx/src/inicio/ArtPdfRed.jsp?iCve=10504907. [Consultado en julio 2011].

CNC -Centro Nacional de Consultoría- (2011). Encuesta: Imagen del presidente Juan Manuel Santos en su sexto mes de gobierno. [En línea] Centro Nacional de Consultoría. Abril 2011. Disponible en http://www.centronacionaldeconsultoria.com/estudios.php. [Consultado en julio 2011].

DANE -Departamento Administrativo Nacional de Estadística- (2010). Guía para Diseño, Construcción e Interpretación de Indicadores. Estrategia para el Fortalecimiento Estadístico Territorial. Herramientas estadísticas para una gestión territorial más efectiva. [En línea]. Bogotá D.C. 42p. Disponible en http : // www.dane.gov.co / files / planificacion / fortalecimiento / cuadernillo/Guia construccion interpretacion indicadores.pdf. [Consultado en abril 2011].

DNP -Departamento Nacional de Planeación- (2008). Guía para elaboración de indicadores. [En línea]. Bogotá D. C. Grupo Asesor de la Gestión de Programas y Proyectos de Inversión Pública- GAPI E SINERGIA. 20p. Disponible en http: // www.dnp.gov.co / PortalWeb / Portals / 0 / archivos / documentos / DIFP / Bpin / Guia para elaboracion de in dicadores.pdf. [Consultado en mayo 2011]

CRC - Congreso de la República de Colombia- (1992). Decreto 2130 de 1992. Por el cual se atribuyen funciones a los Ministros, Directores de Departamentos Administrativos y demás directores, gerentes o jefes de entidades administrativas del orden nacional. Bogotá D. C. Diario Oficial No. 40703, del 31 de diciembre de 1992.

CRC -Congreso de la República de Colombia- (1996). Decreto 111 de 1996. Por el cual se compilan la Ley 38 de 1989, la Ley 179 de 1994 y la Ley 225 de 1995 que conforman el estatuto orgánico del presupuesto. Bogotá D. C. Diario Oficial 42.692 de enero 18 de 1996.

CRC - Congreso de la República de Colombia- (1993). Ley 80 de 1993. Por la cual se expide el Estatuto General de Contratación de la Administración Pública. Bogotá D. C. Diario Oficial N. 41.094 de 28 de octubre de 1993.

CRC -Congreso de la República de Colombia- (1998). Ley 489 de 1998. Por la cual se dictan normas sobre la organización y funcionamiento de las entidades del orden nacional, se expiden las disposiciones, principios y reglas generales para el ejercicio de las atribuciones previstas en los numerales 
Encuesta de opinión para la evaluación de la gestión pública en Colombia: una propuesta de medición

15 y 16 del artículo 189 de la Constitución Política y se dictan otras disposiciones. Bogotá D. C. Diario Oficial N. 43.464, de 30 de diciembre de 1998.

CRC -Congreso de la República de Colombia- (2007). Ley 1150 de 2007. Por medio de la cual se introducen medidas para la eficiencia y la transparencia en la Ley 80 de 1993 y se dictan otras disposiciones generales sobre la contratación con Recursos Públicos. Bogotá D. C. Diario Oficial N. ${ }^{\circ} 46.691$ de 16 de julio de 2007.

FMI -Fondo Monetario Internacional- (2008). Manual de transparencia fiscal. [En línea]. Washington, D.C. 179p. Disponible en línea en http://www.imf.org/external/np/fad/trans/spa/ manuals.pdf. [Consultado en marzo de 2011]

FMI -Fondo Monetario Internacional- (2006). Indicadores de solidez financiera. Guía de compilación. [En línea]. Washington, D. C. 328p. Disponible en línea en http://www.imf.org. [Consultado en agosto de 2011]

FMI -Fondo Monetario Internacional- (2001). Manual de estadísticas de finanzas públicas. [En línea]. Washington, D.C. 173p. Disponible en línea en http://www.imf.org/external/pubs/ ft/gfs/manual/esl/index.htm. [Consultado en mayo de 2011]

FMI -Fondo Monetario Internacional- (1997). Good Governance. The IMF's Role. [En línea]. Washington, D. C. 20p. Disponible en Internet en http://www.imf.org/external/pubs/ft/ exrp/govern/govindex.htm. [Consultado en mayo de 2011]

Gallup Colombia (2011). Encuesta Gallup Poll Bimestral 82. [En línea]. En: Portal Internet la Silla Vacía. Bogotá D.C. Publicado en Mayo de 2011. 120p. Disponible en internet en: http:// www.lasillavacia.com/sites/default/files/media/photo/24556/gallup.pdf. [Recuperado Junio de 2011].

Guerrero, Juan Pablo (1995). La evaluación de las políticas públicas, enfoques teóricos y realidades en nueve países desarrollados. [En línea]. Gestión y Política Pública. Vol. IV. No. I. Primer semestre de 1995. pp. 47-115. Disponible en linea en http://www.gestionypoliticapublica.cide.edu/num anteriores/Vol.IV No.I lersem/GAJ Vol.4 No.I $1 \mathrm{sem}$. pdf. [Recuperado Junio de 2011].

BM -Banco Mundial- y CNC (2002). Encuesta: corrupción, desempeño institucional y gobernabilidad: desarrollando una estrategia anti-corrupción para Colombia. [En línea]. Diagnóstico acerca de la corrupción y gobernabilidad en Colombia. Bogotá D. C. Febrero 7 de 2002. Disponible en http://info.worldbank.org/etools/docs/library/206626/col informefinal2002.pdf. 56p. [Recuperado en Junio de 2011]

INF -Ipsos Napoleón Franco- (2009). La Gran Encuesta 2010- Medición 1. [En línea]. En Documentos Semana.com. Bogotá D.C. Junio 1 de 2009 - Estudio N. ${ }^{\circ} 1850$. 57p. Disponible en: http://www.semana.com/documents/Doc-1886 200961.pdf. [Recuperado en Agosto de 2011]. 
Henry Antonio Mendoza Tolosa • William Orlando Prieto Bustos • Carlos Alberto Barreto Nieto

Lahera, P. Eugenio. (2004). [En línea]. Política y políticas públicas, Serie 95. Santiago de Chile. CEPAL. Agosto de 2004. LC/L.2176-P. Nº denta: S.04.II.G.103. Disponible en línea en http://tramites.jalisco.gob.mx/images/politicaspublicas.pdf. [Recuperado en Abril de 2011].

UNDEF -Fondo de las Naciones Unidas para la Democracia- (2010). Encuesta: Percepción electoral de los votantes colombianos. Misión de Observación Electoral (MOE). [En línea]. Bogotá D.C. Publicado en Enero 2011. Disponible en: http://moe.org.co/home/doc/encuesta/ encuestamoe.pdf. [Recuperado en Junio de 2011]

Ospina Bossi, Sonia. (2000). Evaluación de la gestión pública: conceptos y aplicaciones en el caso latinoamericano. [En línea]. V Congreso Internacional del CLAD sobre la Reforma del Estado y de la Administración Pública, Santo Domingo, Rep. Dominicana, 24-27 Oct. 2000. 23p. Disponible en línea en http://unpanl.un.org/intradoc/groups/public/documents/CLAD/clad0038514.pdf. [Recuperado el 09 de Abril de 2011].

Prieto William, Barreto Carlos y Mendoza Henry (2011). La eficiencia del sector público. Documento de trabajo. Universidad Católica de Colombia, Facultad de Economía, Grupo de investigación Política Económica. p. 28.

Torres S, Gerardo. (2007). La evaluación de políticas y programas públicos. El caso de los programas de desarrollo social en México. [En línea]. En: Revista Política y Cultura. No. 27. Redalyc. Universidad Autónoma Metropolitana. México. pp. 197-201. Disponible en línea en http://redalyc.uaemex.mx/src/inicio/ArtPdfRed.jsp?iCve=26702710. [Recuperado en Septiembre de 2011]. 


\section{Anexo}

\section{ENCUESTA DE GESTIÓN DEL EJECUTIVO-EGE}

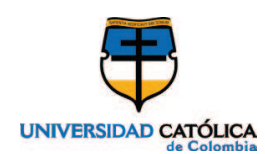

\section{a. Ejecución presupuestal}

Justificación de la pregunta: Evaluar la efectividad de la gestión presupuestal del Ministerio XYZ de conformidad con las responsabilidades asignadas.

1. ¿En relación con lo establecido en el Plan Financiero correspondiente a la vigencia fiscal actual, considera que la ejecución del presupuesto a cargo del Ministerio XYZ durante el trimestre (Ti) fue?

\begin{tabular}{|l|l|}
\hline No adecuada & \\
\hline Relativamente adecuada & \\
\hline Adecuada & \\
\hline
\end{tabular}

Argumentación:

\section{b. Cumplimiento de metas}

Justificación de la pregunta: Evaluar el avance de las metas gubernamentales propuestas por el ejecutivo por sector específico durante el periodo analizado, con base en indicadores específicos a los cuales el Departamento Nacional de Planeación les hace seguimiento.

2. ¿Según lo establecido en el Plan Nacional de Desarrollo, el avance en las metas formuladas para el Sector XYZ por parte del Ministerio XYZ durante el trimestre (Ti) fue?

\begin{tabular}{|l|l|}
\hline No adecuado & \\
\hline Relativamente adecuado & \\
\hline Adecuado & \\
\hline
\end{tabular}

Argumentación: 


\section{c. Contratación:}

Justificación de la pregunta: Evaluar la transparencia de los procesos de contratación en cada cartera así como el avance de los productos y servicios contratados.

3. ¿De conformidad lo establecido en el estatuto general de la contratación pública (Ley 80 de 1993, Ley 1150 de 2007) los procesos de contratación a cargo del Ministerio XYZ durante el trimestre (Ti) se adelantaron de manera?

\begin{tabular}{|l|l|}
\hline $\begin{array}{l}\text { No avanzaron en forma } \\
\text { adecuada }\end{array}$ & \\
\hline $\begin{array}{l}\text { Avanzaron en forma } \\
\text { relativamente adecuada }\end{array}$ & \\
\hline Avanzaron en forma adecuada & \\
\hline
\end{tabular}

Argumentación:

Justificación de la pregunta: Evaluar el avance de los productos y servicios contratados.

4. ¿Durante el trimestre (Ti), de conformidad lo establecido en el estatuto general de la contratación pública (Ley 80 de 1993, Ley 1150 de 2007) y los programas contemplados en el Plan Nacional de Desarrollo, los bienes y servicios contratados por el Ministerio XYZ?

\begin{tabular}{|l|l|}
\hline $\begin{array}{l}\text { Un efecto negativo para la } \\
\text { sociedad }\end{array}$ & \\
\hline $\begin{array}{l}\text { Fueron indiferentes para la } \\
\text { sociedad }\end{array}$ & \\
\hline $\begin{array}{l}\text { Un efecto positivo para la } \\
\text { sociedad }\end{array}$ & \\
\hline
\end{tabular}

Argumentación:

\section{d. Impacto en la sociedad de las medidas}

Justificación de la pregunta: Determinar con objetividad el impacto social comunidad con independencia de la percepción general de las personas.

5. ¿Durante el trimestre (Ti), las medidas y adelantadas por el Ministerio XYZ representaron?

\begin{tabular}{|l|l|}
\hline $\begin{array}{l}\text { Un efecto negativo para la } \\
\text { sociedad }\end{array}$ & \\
\hline $\begin{array}{l}\text { Fueron indiferentes para la } \\
\text { sociedad }\end{array}$ & \\
\hline $\begin{array}{l}\text { Un efecto positivo para la } \\
\text { sociedad }\end{array}$ & \\
\hline
\end{tabular}


Encuesta de opinión para la evaluación de la gestión pública en Colombia: una propuesta de medición

Argumentación:

Justificación de la pregunta: Determinar el grado de aceptación de las medidas de gobierno por parte de los ciudadanos

6. ¿Durante el trimestre (Ti), las medidas y acciones adelantadas por el Ministerio $\mathrm{XYZ}$ ?

No fueron bien recibidas por

las sociedad

Fueron indiferentes para la sociedad

Un efecto positivo para la

sociedad

Argumentación:

\section{e. Eficiencia en la gestión y administración pública}

Justificación de la pregunta: Evaluar la eficiencia de las actividades adelantadas por cada cartera durante el analizado

7. ¿¿Durante el trimestre (Ti), como califica la gestión del Ministerio XYZ?

\begin{tabular}{|l|l|}
\hline Ineficiente & \\
\hline Relativamente eficiente & \\
\hline Eficientes & \\
\hline
\end{tabular}

Argumentación:

\section{f. Evolución del trámite de proyectos de interés}

Justificación de la pregunta: Determinar la oportunidad así como del avance en el trámite de normas relacionadas directamente con las metas y propósitos del gobierno en cada sector. 
8. ¿Durante el trimestre (Ti), el trámite de proyectos de ley y/o expedición de normas relacionadas con las competencias adelantadas por el Ministerio XYZ fue?

\begin{tabular}{|l|l|}
\hline Insatisfactorio & \\
\hline Satisfactorio & \\
\hline $\begin{array}{l}\text { No se presentaron/adelantaron proyectos de } \\
\text { ley y/o normas por parte del Miniterio }\end{array}$ & \\
\hline
\end{tabular}

Argumentación:

\section{g. Relaciones internacionales}

Justificación de la pregunta: Impacto del manejo de las relaciones diplomáticas del país con Estados vecinos y no vecinos.

9. ¿Durante el trimestre (Ti), las actividades adelantadas por el Ministerio XYZ?

\begin{tabular}{|l|l|}
\hline $\begin{array}{l}\text { No fortalecieron las relaciones internacio- } \\
\text { nales del país }\end{array}$ & \\
\hline $\begin{array}{l}\text { Fueron indiferentes para las relaciones inter- } \\
\text { nacionales del país }\end{array}$ & \\
\hline $\begin{array}{l}\text { Fortalecieron las relaciones internacionales } \\
\text { del país }\end{array}$ & \\
\hline
\end{tabular}

Argumentación:

Justificación de la pregunta: En un contexto de apertura y globalización mundial determinar (en que grado) el país es perceptor/prestador neto de cooperación técnica en los temas que son responsabilidad de cada Ministerio.

10. ¿Durante el trimestre (Ti), las actividades adelantadas por el Ministerio XYZ?

No fortalecieron las relaciones de cooperación con otros países en los temas a su cargo Fueron indiferentes en términos de cooperación con otros países en los temas a su cargo Fortalecieron las relaciones de cooperación con otros países en los temas a su cargo

Argumentación: 